\title{
Norois
}

Environnement, aménagement, société

$244 \mid 2017$

Sport, immigration, urbanisation littorale, climat sahélien, biodiversité

\section{Le monde de l'Ovalie et ses espaces}

Les conditions d'entrée dans la pratique de jeunes sportifs

The world of French rugby and its spaces. Young sportspersons'

conditions for entry

Hélène Joncheray, David Sudre et Antoine Lech

\section{(2) OpenEdition}

\section{Édition électronique}

URL : https://journals.openedition.org/norois/6144

DOI : $10.4000 /$ norois. 6144

ISBN : 978-2-7535-7420-5

ISSN : $1760-8546$

Éditeur

Presses universitaires de Rennes

Édition imprimée

Date de publication : 30 décembre 2017

Pagination : 7-17

ISBN : 978-2-7535-7418-2

ISSN : 0029-182X

\section{Référence électronique}

Hélène Joncheray, David Sudre et Antoine Lech, « Le monde de l'Ovalie et ses espaces », Norois [En ligne], 244 | 2017, mis en ligne le 30 décembre 2019, consulté le 14 janvier 2022. URL : http:// journals.openedition.org/norois/6144; DOI : https://doi.org/10.4000/norois.6144 


Nöiels

\title{
Le monde de l'Ovalie et ses espaces Les conditions d'entrée dans la pratique de jeunes sportifs
}

\author{
The World of French Rugby and its Spaces. Young Sportspersons' Conditions for Entry
}

\author{
Hélène JoncheraY ${ }^{\mathrm{a}}$, David Sudre ${ }^{\mathrm{b}}$ et Antoine $\mathrm{LECH}^{\mathrm{c}}$
}

\footnotetext{
${ }^{a}$ Chercheure, INSEP, Laboratoire Sport, Expertise et Performance (EA 7370) et Techniques et Enjeux du Corps (EA 3625), Université Paris Descartes, Sorbonne Paris Cité. (helene.joncheray@parisdescartes.fr)

${ }^{\text {b }}$ Docteur en sociologie, Université Paris Descartes, Sorbonne Paris Cité, Laboratoire Techniques et Enjeux du Corps (TEC, EA 3625). (davidsudre@hotmail.com)

' Docteur en sociologie, Université Paris Descartes, Sorbonne Paris Cité, Laboratoire Techniques et Enjeux du Corps (TEC, EA 3625). (antoine.lech@hotmail.fr)
}

Résumé : En France, la géographie du rugby à XV présente une prégnance forte dans le Sud-Ouest, où les licenciés sont surreprésentés au regard des autres régions. L'article interroge l'influence de cette particularité régionale sur les conditions d'entrée dans la pratique des jeunes joueurs de rugby. En d'autres termes, l'objectif principal de l'article est de saisir les différentes modalités d'accès au rugby au regard de la région de pratique. L'enquête, menée par questionnaires, a permis d'interroger 2455 enfants âgés de 9 à 11 ans. Les résultats montrent que la spécificité géographique du rugby a peu d'influence sur les conditions d'entrée dans la pratique de ces jeunes joueurs. Ainsi, si des différences régionales sont observées, elles ne sont pas liées au caractère dit rugbystique des espaces en question.

\begin{abstract}
In France, rugby union's geography features a pervasiveness in the south-west, where licence-holders are over-represented in comparison to other regions. The article questions the influence of this regional characteristic on young rugby players' conditions for entry. In other words, the paper's main aim is to grasp the different conditions for access to rugby depending on the region one lives in. Carried out through questionnaires, the survey made it possible to interview 2,455 children between the ages of 9 and 11. The results show that rugby's geographical specificity has little influence on these young players' conditions for entry. If some regional differences can be observed, they are not linked to the importance of rugby in these spaces.
\end{abstract}

Mots clés : rugby - jeunesse - socialisation - région - sport

Keywords: rugby union - young players - socialisation - region - sport

\section{INTRODUCTION}

Le rugby à $\mathrm{XV}$, pratique sportive et ludique arrivée d'Angleterre en France au début des années 1870, présente, au sein du monde sportif français, un trait distinctif, dans le sens où il a toujours semblé possé- der, voire revendiquer, des particularités liées à son implantation géographique. Ainsi, son emprise spatiale est reconnue pour être très importante dans le Sud-Ouest de la France. Plusieurs études ont montré que le monde de l'ovalie concentrait, en effet, ses 
effectifs dans la partie méridionale de l'Hexagone. Un «effet midi » renforcerait la coupure de la France en deux ensembles géographiques distincts; de part et d'autre d'une ligne Le Havre-Marseille s'opposeraient une France de l'Ouest à forte pratique et une France de l'Est où elle s'exprimerait avec plus de discrétion (Mathieu et al., 1992).

Toutefois, cela ne signifie pas, pour autant, que la majorité des sportifs licenciés du Sud-Ouest soient des joueurs de rugby. Ce dernier n'est d'ailleurs pas le sport le plus pratiqué dans cette partie de la France. Haumont (1995) précise en effet que le premier rang revient au football, mais que le SudOuest reste la région où le rapport numérique est le plus élevé entre l'effectif des pratiquants de rugby et celui des pratiquants de tous les autres sports. Ces résultats s'expliquent, en partie, par le contraste important du nombre total de licenciés inscrits auprès de ces deux fédérations nationales : le football en compte plus de deux millions, alors que le rugby en comptabilise 450000 (Stat-info, 2014).

En nous intéressant de près à cette pratique ludique et sportive que représente le rugby, nous souhaitons démontrer que le monde de l'ovalie en France ne peut être perçu comme un monde homogène fait par et pour les joueurs du Sud-Ouest, qui se limiterait spatialement à cette région. Au contraire, nous visons à déconstruire certaines idées reçues associées au monde du rugby, telles que la territorialisation de ce sport et son effet sur les plus jeunes pratiquants. Nous faisons l'hypothèse que les régions dites rugbystiques, réputées à «forte pratique » comme le Sud-Ouest, devraient se distinguer des autres par des modalités spécifiques d'accès à la pratique du rugby. $\mathrm{Si}$, au contraire, nous relevons une homogénéité entre les régions, nous pourrons conclure que la socialisation rugbystique s'opère progressivement uniformément en France, et donc que l'ancrage du rugby dans le Sud-Ouest s'estompe peu à peu. Pour procéder à cette analyse comparative, nous retenons les variables habituellement utilisées dans les enquêtes sur la socialisation sportive, comme l'âge d'accès à la pratique sportive et rugbystique, le passé sportif, l'influence de la famille, des amis et de l'école. Enfin, enquêter sur les enfants est particulièrement adapté pour mesurer ce phénomène, puisque l'environnement social, culturel et familial exerce une influence considérable à cet âge de la vie. L'effet normatif du contexte est d'autant plus saillant et observable.
Avant d'exposer les résultats de notre travail, nous aborderons les recherches effectuées sur le sujet et présenterons la méthodologie choisie. Nous définirons ensuite, par rapport aux régions, les caractéristiques sociales des jeunes joueurs de rugby en lien avec le contexte dans lequel se produit leur engagement dans la pratique sportive et rugbystique.

\section{Le Sud-Ouest et sa " mainmise » sur le rugby français}

Plusieurs explications sont proposées pour répondre à la question de l'implantation du rugby dans le Sud-Ouest. Augustin $(1990,2007)$ et Callède $(1993,1996)$ ont montré que des facteurs d'ordre idéologique et politique se sont superposés d'emblée à la diffusion géographique du ballon ovale et du ballon rond. Si, dans le Sud-Ouest, les instituteurs laïcs adoptent le rugby, c'est le football qui, dans la France du Nord, devient rapidement l'apanage des patronages catholiques qui encadrent la jeunesse. Augustin et Joncheray (2007) précisent que « si le rugby a pu se développer dans le SudOuest, c'est (également) pour des raisons institutionnelles et du fait de son antécédence à tout autre sport emblématique » Augustin (1990, 2004). avance trois raisons pour expliquer l'implantation du rugby dans cette région : la présence d'une colonie britannique dans les milieux d'affaires bordelais, la place de la Ligue girondine d'éducation physique et, enfin, le succès sportif du Stade Bordelais.

Soulignons également que, pendant longtemps, les clubs de haut niveau étaient justement localisés dans les zones de forte implantation rugbystique et réciproquement (Mathieu, Praicheux, 1987 ; Ravenel, 1998, 2000, 2004 ; voir figure 1). Aujourd'hui encore, le rugby à XV reste fortement présent dans les villes du Sud, notamment dans les villes moyennes où il participe de la vie socio-économique (Augustin, 2007). Ainsi, depuis les années 1920, le rugby à XV semble donc conserver, de façon prononcée, son caractère régional, là où les autres pratiques sportives, à l'image du football, voient leur développement se propager de façon plus homogène sur l'ensemble du territoire français (Ravenel, 1998, 2000; Wahl, 1989).

Toutefois, la professionnalisation du rugby, actée en 1995, atténue progressivement en France son ancrage dans le Sud-Ouest. L'afflux de joueurs 
étrangers, l'émergence de clubs performants dans des grandes villes situées en dehors de sa zone historique d'implantation (Clermont-Ferrand, Lyon et Toulon notamment) et leurs succès sportifs ont fait du Top 14 l'un des championnats les plus compétitifs et suivis au monde (diffusé dans plus de 190 pays).

Cet article entend analyser, pour les jeunes générations, les conditions d'entrée dans la pratique dans ce nouveau contexte où le rugby tend à se globaliser et se rapprocher du modèle économique et organisationnel du sport-spectacle (Augustin, 2007). Observe-t-on toujours des différences inter-régionales importantes, ou au contraire une uniformisation au niveau national des modalités d'entrée dans la pratique? Il ne s'agit pas de remettre en cause l'existence d'une « terre de rugby » (Darbon, 1997; Ravenel, 2004), mais plutôt de sonder ses nouvelles frontières (Gay, 2006) : peutêtre diluées géographiquement mais tout de même fortes symboliquement.

\section{Le monde rugbystique : un espace de sociabilité spécifique}

Au regard de l'implantation spécifique du rugby à $\mathrm{XV}$, nous nous demandons si l'engagement des jeunes joueurs dans cette pratique est influencé par cet espace de sociabilité rugbystique (Augustin et Garrigou, 1985; Darbon, 1997) et par conséquent si le mode d'entrée dans la pratique des joueurs est différent en fonction de leur région. Tout en tenant compte des processus socio-historiques et des réseaux sociaux, Augustin (2007) explique qu'à travers la notion de territorialité, « le discours sur la jeunesse est recodé et le sport, après avoir été révélateur des rivalités, s'affirme comme un vecteur d'identité, chaque sport constituant alors un pôle privilégié d'identification communautaire » (Augustin, 2007, p. 64; Tonini, 2012). Si le monde social du rugby est « lié à des lieux spécifiques » (Strauss, 1978, p. 273), nous posons la question de son influence sur les modes d'entrée dans la pratique des jeunes joueurs.

Si l'enquête découvre que l'entrée dans la pratique rugbystique s'opère de la même manière dans le SudOuest que dans le reste de la France, alors le monde de l'ovalie peut être pleinement défini comme un monde social (Becker, 2006), dans la mesure où l'apprentissage des conventions appropriées pour pouvoir y participer emprunte les mêmes chemins quelle que

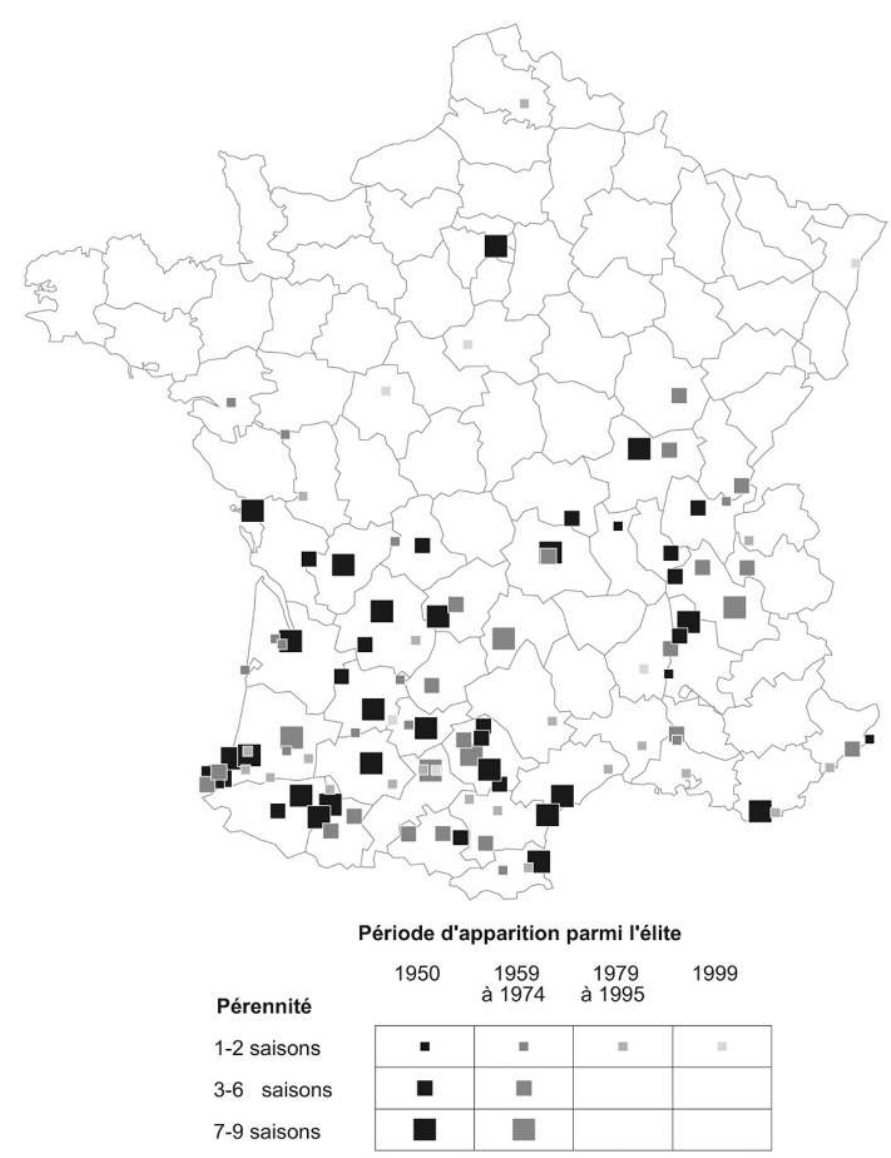

Figure 1 : La diffusion du rugby de haut niveau (1950-1999) (Source : Ravenel, 2004)

The distribution of high-level rugby (1950-1999)

soit la région considérée. En effet, comme le précise Becker, le monde social désigne moins une unité de chose ou une entité spatiale close (Becker, 1988) qu'une activité collective sur un espace extensible dont font partie tous les acteurs qui contribuent à le faire vivre : aussi bien ceux qui œuvrent à sa production que ceux qui participent de sa diffusion et de sa consommation (Becker, Pessin, 2006). C'est pourquoi nous allons analyser attentivement l'influence exercée par les parents, les clubs sportifs et l'école sur les jeunes pratiquants du rugby, afin de mesurer la socialisation sportive de ces derniers selon leur région de pratique.

\section{La socialisation sportive des jeunes enfants}

La socialisation est le « processus par lequel les individus apprennent des habiletés, valeurs, atti- 
tudes, normes et connaissances associées à un rôle social » (McPherson, Brown, 1988 : 267). Selon Bois et Sarrazin (2006, p. 12), trois types de socialisations sportives sont distingués par Brustad (1992) et Greendorfer (1992) : « une socialisation à faire du sport (socialization into sport) qui renvoie aux processus amenant un individu à pratiquer et à rester investi dans une activité physique; une socialisation par le sport (socialization through sport) qui désigne les processus entraînant le développement de traits moraux ou psychologiques par la pratique sportive; et la socialisation conduisant à l'arrêt de la pratique sportive (socialization out of sport) qui fait référence aux processus d'influence sociale pouvant être néfastes à la pratique sportive ». Nous nous intéressons principalement au premier type de socialisation évoqué et notamment au rôle de la famille, celle-ci étant reconnue comme ayant une influence décisive sur le choix de pratique sportive des enfants et des adolescents.

Au sein de ce mode de socialisation à faire du sport, nous cherchons donc à mieux connaître les modalités d'entrée des jeunes joueurs dans la pratique du rugby et à dévoiler, si elles existent, des différences en fonction des régions. Nous cherchons donc à savoir si l'implantation historique du rugby dans le Sud-Ouest de la France agit sur les facteurs d'introduction à cette pratique sportive.

\section{MÉTHOde : OUTILS ET ÉCHANTILLON}

Les travaux ont été réalisés en collaboration avec la cellule recherche de la Fédération française de rugby (FFR). L'un des auteurs de l'article coopère depuis plusieurs années avec la FFR, sur différentes enquêtes. De concert avec sa Direction technique nationale adjointe, axée sur le développement du rugby, plusieurs réunions ont permis d'identifier des questionnements sur les conditions d'entrée dans la pratique des jeunes joueurs de rugby, au regard de leurs régions de pratique. Ces interrogations ont été discutées puis mises en forme tout au long des pré-enquêtes et de l'enquête finale.

\section{Outils : des pré-enquêtes au questionnaire final}

Trois pré-enquêtes ont permis de construire le questionnaire définitif. Dès la première pré-enquête et dans la perspective de l'enquête finale, le choix a été fait d'administrer indirectement le questionnaire auprès des joueurs, via les éducateurs de clubs sportifs. Dans la mesure où la population étudiée est composée exclusivement d'enfants, procéder ainsi permettait d'avoir leur confiance, celle des parents et de s'assurer un maximum de répondants.

La première pré-enquête a permis d'interroger 74 joueurs de 11 ans, par l'intermédiaire de cinq questions ouvertes, posées oralement par leur éducateur : "pourquoi joues-tu au rugby; qu'est-ce que tu aimes dans le rugby; qu'est-ce que tu préfères; qu'est-ce que tu n'aimes pas; qu'est-ce que tu détestes? » Le choix de questions ouvertes a permis d'obtenir une connaissance plus précise du vocabulaire utilisé par les enfants de cet âge. Cette première pré-enquête a confirmé le fait que les enfants rencontraient des difficultés à expliquer réellement pourquoi ils jouaient au rugby et que les dernières questions tournées autour d'éléments négatifs suscitaient moins de réponses. Cette pré-enquête a succédé à l'observation d'un tournoi d'une demijournée, opposant des équipes issues de trois clubs.

Une deuxième pré-enquête a été réalisée auprès d'un échantillon restreint de 10 joueurs, au sein d'un club situé en Île-de-France. 17 questions fermées étaient posées. Malgré la crainte justifiée que nous pouvions avoir - les enfants ont du mal à expliquer, à verbaliser - nous avons constaté que, grâce à la première pré-enquête réalisée et au vocabulaire relevé, la formulation des questions a permis aux jeunes joueurs de bien les comprendre et de donner des réponses variées.

La troisième et dernière pré-enquête a permis de tester un questionnaire de 18 questions à l'échelle du comité de Franche-Comté. Deux journées de tournoi ont permis de récolter 151 questionnaires.

Le questionnaire définitif, construit par l'intermédiaire de ces trois pré-enquêtes, était composé de 20 questions dont cinq ouvertes. Différents thèmes étaient abordés : le passé sportif des jeunes joueurs, l'arrivée à la pratique du rugby, les influences familiale et scolaire. Le questionnaire a été diffusé, nationalement, par l'intermédiaire des conseillers techniques territoriaux qui les ont ensuite transmis aux éducateurs des clubs de rugby. Ces derniers ont donc eu en charge la passation, la récolte puis le retour des questionnaires. Dans le courrier envoyé avec le questionnaire, de courtes consignes à lire 
précisaient la raison de l'enquête et le mode de fonctionnement à respecter. Ces consignes devaient être lues aux joueurs avant la passation des questionnaires en fin ou en début d'entraînement.

\section{Échantillon : les joueurs interrogés}

Les joueurs interrogés sont licenciés à la Fédération française de rugby et pratiquent le rugby de façon organisée dans des clubs, dans la catégorie d'âge des 9-11 ans.

Sur les 450000 licenciés recensés à la Fédération française de rugby, 25000 sont des joueurs inscrits dans la catégorie d'âge de 9 à 11 ans. Parmi ces 25000 joueurs, tous sollicités, 2700 d'entre eux ont répondu au questionnaire. Presqu'un dixième de la population a donc pu être interrogé. Sur l'ensemble des questionnaires récoltés, 2455 ont été exploités, représentant 169 clubs et 18 des 22 régions que compte la France métropolitaine. Faute de ne pas avoir eu de joueurs de rugby ayant répondu à l'enquête, quatre régions - Champagne-Ardenne, Limousin, Corse et Lorraine - ne sont pas représentées.

Notre échantillon est représentatif de la répartition à l'échelle nationale de l'ensemble des joueurs de 9 à 11 ans inscrits auprès de la Fédération française de rugby. Si l'on scinde la France en deux parties selon une ligne imaginaire passant par le Poitou-Charentes, le Limousin, l'Auvergne et le Rhône-Alpes (Mathieu et al., 1992 ; Ravenel, 2004), $35 \%$ des joueurs interrogés sont licenciés au Nord et $65 \%$ au Sud; ce qui est assez proche des statistiques globales de la FFR, lesquelles sont respectivement de $44 \%$ et $56 \%$.

\begin{tabular}{|c|c|c|c|c|}
\hline Âge & 9 ans & 10 ans & 11 ans & Total \\
\hline Nord & $21,3 \%$ & $56,3 \%$ & $22,4 \%$ & $100 \%$ \\
\hline Sud & $20,9 \%$ & $54,6 \%$ & $24,5 \%$ & $100 \%$ \\
\hline Total & $21 \%$ & $55,2 \%$ & $23,8 \%$ & $100 \%$ \\
\hline
\end{tabular}

Tableau 1 : Distribution des joueurs en fonction de l'âge, selon une répartition Nord/Sud $(\mathrm{p}<0.05)$

The distribution of players according to their age, based on a north/south division $(p<0.05)$

L'analyse statistique s'opère sur trois échelles distinctes : à l'échelle des régions; à l'échelle d'espaces régionaux plus conséquents : Nord et Sud; mais aussi à l'échelle de cinq zones géographiques afin de permettre la comparaison entre des territoires considérés comme rugbystiques et d'autres qui le sont moins, voire pas du tout (voir figure 2). Nous avons délimité ces cinq zones en considérant le nombre de clubs professionnels et de joueurs licenciés (Mathieu et al., 1992 ; Ravenel, 2004), mais aussi le nombre de répondants suffisant pour le traitement statistique de notre étude.

Nous obtenons ainsi cinq zones aux profils variés : a) la zone historique d'implantation du rugby : le « SudOuest » (Aquitaine et Midi-Pyrénées); b) les zones rugbystiques (développées ou structurées) : le «SudEst » (Rhône-Alpes et Provence-Alpes-Côte-D'azur) et l'« Île de France »; et enfin, c) les zones non considérées comme rugbystiques : l'« Ouest » (Bretagne, Paysde-la-Loire et Poitou-Charentes) et le « Nord » (HauteNormandie, Picardie et Nord-Pas-de-Calais). Pour ces cinq zones géographiques, nous obtenons, au regard de notre échantillon, une répartition des joueurs, par âge, sensiblement identique (tableau 2) à celle de la répartition Nord/Sud (tableau 1).

\begin{tabular}{|c|c|c|c|c|}
\hline Âge & 9 ans & 10 ans & 11 ans & Total \\
\hline Nord & $19,1 \%$ & $56,6 \%$ & $24,3 \%$ & $100 \%$ \\
\hline Île-de-France & $21,5 \%$ & $54,1 \%$ & $24,4 \%$ & $100 \%$ \\
\hline Ouest & $22,6 \%$ & $56,6 \%$ & $20,8 \%$ & $100 \%$ \\
\hline Sud-Ouest & $19,6 \%$ & $54,1 \%$ & $26,4 \%$ & $100 \%$ \\
\hline Sud-Est & $20,7 \%$ & $55,2 \%$ & $24,1 \%$ & $100 \%$ \\
\hline Total & $20,9 \%$ & $55.2 \%$ & $23,9 \%$ & $100 \%$ \\
\hline
\end{tabular}

Tableau 2 : Distribution des joueurs en fonction de l'âge, selon une répartition par zone géographique $(\mathrm{p}<0.05)$

The distribution of players in geographical areas according to their age $(p<0.05)$

\section{RÉSULTATS ET DISCUSSION : LES CONDITIONS D'ENTRÉE DANS LA PRATIQUE DU RUGBY À L'ÉPREUVE DU MONDE RUGBYSTIQUE}

Les résultats étudient la socialisation sportive chez des pratiquants de rugby âgés de 9 à 11 ans. Ils ont été traités par l'intermédiaire du logiciel Sphinx ${ }^{2}$, en respectant le seuil de signification statistique de $5 \%$.

Afin de saisir les modalités d'accès à la pratique rugbystique en fonction des régions, nous avons d'abord décidé de comparer l'âge moyen des enfants au moment de leur inscription dans un club sportif, puis de mesurer l'influence exercée par leur entourage, leur famille et l'école dans le choix du rugby. 


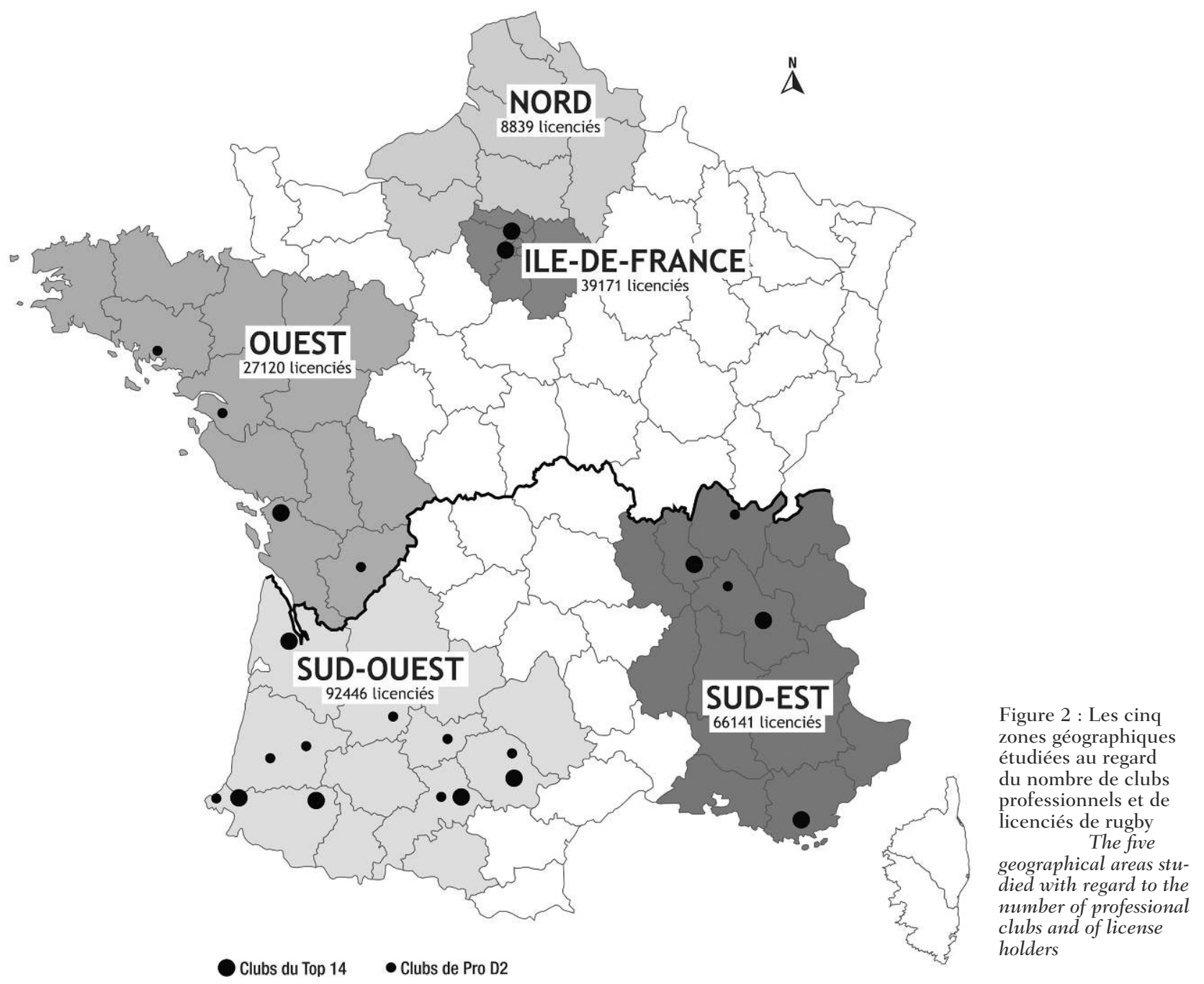

Observe-t-on des spécificités régionales persistantes, qui continuent de distinguer les «terres de rugby » des autres, ou au contraire une tendance à l'uniformisation à l'échelle nationale? C'est par la comparaison que nous pourrons saisir les contours des territoires labélisés rugbystiques et évaluer leur prégnance dans la socialisation sportive des plus jeunes.

\section{L'âge de la première inscription dans un club de sport et dans un club de rugby}

\section{Un accès à la pratique sportive plus tardif au Nord de la Loire}

Avant de débuter le rugby, $70 \%$ des enfants interrogés avaient déjà pratiqué un autre sport.
La moyenne d'âge d'accès à ce premier sport est de 6,7 ans. L'âge d'accès est significativement plus précoce pour les enfants habitant dans le Sud de la France (6,4 ans) que pour ceux du Nord (7,2 ans). De façon significative, les résultats montrent que l'accès à la pratique sportive en club est plus tardif pour les enfants habitant au nord de la Loire que pour ceux du Sud.

\section{L'âge de la première licence de rugby: des différences régionales}

S'ils sont une proportion identique sur tout le territoire à avoir pratiqué un autre sport avant de choisir le rugby, c'est globalement un an plus tard que les enquêtés prendront leur première licence de rugby, 
à 7,6 ans en moyenne. Les résultats de notre étude menée sur les cinq zones montrent que les zones « Nord » $(8,13$ ans $)$ et « Ouest » $(7,8$ ans $)$ sont celles où les joueurs accèdent plus tardivement à la pratique du rugby, comparativement aux autres zones.

La moyenne d'âge la plus élevée du début de la pratique du rugby est de 8,3 ans en Picardie contre 7,3 ans pour la moins élevée en PACA. Ce résultat, significatif, souligne, à l'échelle de deux régions un accès privilégié - c'est-à-dire plus précoce - au rugby pour les jeunes évoluant dans une région dite rugbystique. Toutefois, ce résultat doit être nuancé. En effet, concernant la première inscription en club, toutes pratiques sportives confondues, la moyenne la moins élevée d'âge à l'inscription en club est de 6 ans en PACA. Ainsi, alors que nous pourrions être tentés de justifier l'accès précoce au rugby en PACA (7,3 ans) par la prégnance du rugby dans cette région, nous constatons que le PACA est également une région où les jeunes interrogés débutent la pratique sportive prématurément (6 ans), par rapport au reste de l'échantillon. Ces différences peuvent donc être expliquées, non pas par le caractère rugbystique de la région mais par un accès plus précoce à la pratique sportive en général. Le rugby étant bien souvent un deuxième choix de pratique sportive, il est logique que les jeunes socialisés tôt au sport en général s'engagent plus rapidement dans cette pratique.

\section{Filles et garçons : des différences accentuées dans l'âge d'accès à la pratique du rugby}

Alors que l'accès à la pratique sportive se fait sensiblement au même âge chez les filles et les garçons interrogés (6,7 ans), les résultats montrent que les filles débutent beaucoup plus tardivement le rugby en club que les garçons. Ainsi, alors que les enfants de notre échantillon prennent, en moyenne, leur première licence rugbystique à 7,6 ans - ce qui correspond aussi à l'âge moyen auquel les garçons sont inscrits au rugby -, les filles le font un peu plus d'un an après soit à 8,8 ans. Ce résultat peut être associé à la présence de freins sociaux toujours présents quant à la pratique par des filles et/ou des femmes d'un sport étiqueté masculin (Joncheray et al., 2016; Joncheray, Laporte, Tenèze, 2015 ; Joncheray et Tlili, 2013; Mennesson, 2005).

\section{Le passé sportif : judokas et footballeurs, futurs rugbymen}

Deux-tiers des enfants interrogés ont pratiqué par le passé du football ou du judo. Les taux de pratique du football et du judo dans l'échantillon sont similaires (33\% chacun). Toutefois, nous notons, compte tenu des chiffres des licenciés de la Fédération française de football et de la Fédération française de judo, un indice de pénétration beaucoup plus élevé des judokas. En effet, alors que le judo compte quatre fois moins de licenciés, nous en recensons un pourcentage similaire à celui des joueurs de football.

Ainsi, avant d'accéder à un club de rugby, les joueurs ont, en moyenne, pratiqué pendant une année, une autre activité sportive. Et, les garçons ayant été judokas sont plus susceptibles que les autres de choisir une licence de rugby.

Le tableau 3 montre que le rugby constitue majoritairement un deuxième choix de pratique sportive à l'échelle nationale. Non seulement l'étude statistique ne relève aucune différence significative entre les régions rugbystiques et non-rugbystiques, mais plus étonnant encore : c'est dans une zone pourtant peu rugbystique (la zone « Ouest») que l'on observe le plus grand ratio de joueurs ayant opté pour le rugby comme premier choix de pratique sportive.

Ầ ce stade, les facteurs de socialisation rugbystique ne sont pas spécifiques à une zone géographique, ils influencent les jeunes de manière quasiment identique sur l'ensemble du territoire national, car peu de différences inter-régionales ont été relevées.

À cet âge, 9 à 11 ans, l'établissement des relations sociales nécessaires pour construire ce monde s'opère par l'intermédiaire des parents, des clubs et des écoles.

\begin{tabular}{|c|c|c|c|}
\hline Pratique d'un sport avant le rugby & Oui & Non & Total \\
\hline Nord & $74,6 \%$ & $25,4 \%$ & $100 \%$ \\
\hline Île-de-France & $71,1 \%$ & $28,9 \%$ & $100 \%$ \\
\hline Ouest & $65,7 \%$ & $34,3 \%$ & $100 \%$ \\
\hline Sud-ouest & $69,9 \%$ & $30,1 \%$ & $100 \%$ \\
\hline Sud-est & $67,0 \%$ & $33,0 \%$ & $100 \%$ \\
\hline Total & $68,4 \%$ & $31,6 \%$ & $100 \%$ \\
\hline
\end{tabular}

Tableau 3 : La pratique d'un sport avant la pratique du rugby, selon l'appartenance régionale $(\mathrm{p}<0.05)$

Other sports practiced before rugby, according to geographical areas $(p<0.05)$ 


\section{L'accès à la pratique sportive, sous influences familiale et scolaire}

Les joueurs ont été interrogés à un âge où la prise d'une licence implique nécessairement la famille de l'enfant. De nombreuses études sociologiques montrent le lien entre la famille et l'accès des enfants à une pratique sportive : « Le rôle de la famille comme l'élément initial majeur de l'influence sur l'activité physique des enfants semble avéré » (Brustad, 1993, p. 211). Ce rôle, assumé (Lewko et Greendorfer, 1988), vient de l'idée selon laquelle la majorité des parents pense qu'un sport organisé est nécessaire pour leur enfant car il lui permettrait de devenir une personne pleinement développée et mettrait en avant des indicateurs mesurables de son développement (Coakley, Pike, 2009; Fuchs, Augustin, 2014).

\section{Une influence familiale indéniable}

À l'échelle nationale, les résultats mettent en avant un processus de transmission familiale important : $70 \%$ des enquêtés disent avoir été influencés principalement par leur famille pour pratiquer le rugby. L'influence de l'entourage - famille et amis (82\%) peut s'expliquer par le jeune âge des joueurs interrogés, âge auquel les parents choisissent souvent l'activité sportive de leur enfant. On peut imaginer que le résultat aurait été différent chez une population plus âgée, l'influence des parents décroissant avec l'ancienneté rugbystique (Joncheray, Laporte, Tenèze, 2015). Ces résultats rappellent l'importance de la présocialisation (Young, 1999) familiale (Brustad, Babkes, Smith, 2001 ; Coakley, White, 1992), facilitant l'engagement dans une pratique, faisant passer un individu de novice à pratiquant.

Si notre étude statistique confirme que l'influence familiale est belle et bien prépondérante dans la socialisation sportive (Bois, Sarrazin, 2006; Coakley, Pike, 2009), elle montre surtout qu'elle s'exerce uniformément selon les régions. En effet, les familles des zones rugbystiques (Sud-Ouest, Sud-Est et Îlede-France) n'orientent pas davantage leurs enfants vers le rugby que les familles des zones non-rugbystiques (Nord et Ouest), dans la mesure où nous n’avons relevé aucune différence significative quelle que soit l'échelle d'analyse.

\section{L'élément déclencheur : la famille, le club, l'école ou les amis?}

À l'échelle régionale, la transmission par la famille est significativement moins importante en PoitouCharentes; l'influence de l'école est significativement plus importante en Midi-Pyrénées (6,9\%), dans le Nord-Pas-de-Calais $(6,7 \%)$ et en Haute Normandie $(5,1 \%)$ que dans les autres régions; enfin, les amis sont davantage cités, et ce de façon significative, en Haute-Normandie (30,8\%). Au regard de l'ensemble des régions étudiées et vis-à-vis des éléments de réponse proposés, les différences régionales évoquées ci-dessus sont les seules relevées significativement parlant. Elles montrent que, malgré des similarités conséquentes quant à l'accès à la pratique du rugby, l'élément qui, selon les enfants, a déclenché leur pratique du rugby varie en fonction des régions. L'influence, plus conséquente, du réseau amical dans les régions de la HauteNormandie $(30,8 \%)$, des Pays de la Loire $(18,9 \%)$ et de la Picardie $(17,7 \%)$ peut s'expliquer par une plus faible pratique familiale dans ces régions qualifiées de peu rugbystiques. Les autres résultats présentent des différences régionales mais ne s'opposent pas massivement en fonction du caractère rugbystique ou non des territoires en question.

\section{L'école, marqueur d'une identité régionale}

Comme le montre le tableau 4, la « zone Île-deFrance » concède un pourcentage significativement inférieur à l'effectif théorique au sujet de l'école élémentaire $(1,3 \%)$ comme élément déclencheur de la pratique. Ce résultat nous amène à affirmer l'importance de l'institution scolaire favorisant des différences culturelles sportives. C'est ce que montrent les réponses des jeunes joueurs interrogés, l'accès à la pratique du rugby par l'école est significativement plus élevé dans la «zone Nord» $(5,1 \%)$ que dans la « zone Sud-est » $(1,5 \%)$ et dans la « zone Île-deFrance $»(1,3 \%)$.

Quant à la pratique du rugby à l'école, l'Aquitaine $(57,8 \%)$ est la région où les enfants s'y adonnent le plus alors qu'en Bretagne beaucoup moins (22,9\%). Ce sont les seules différences relevées à l'échelle régionale. Ces différences s'affirment également entre le Nord et le Sud, les enfants jouant ou ayant joué au rugby à l'école dans le Nord de la France 
Tableau 4 : L'élément déclencheur quant au choix de la pratique, en fonction d'une différenciation par zone géographique $(\mathrm{p}<0.05)$

The event that triggered the decision to play rugby, according to geographical areas $(p<0.05)$

\begin{tabular}{|c|c|c|c|c|c|c|}
\hline Élément déclencheur & Famille & Club & École & Amis & Autre & Total \\
\hline Nord & $64,2 \%$ & $0,7 \%$ & $5,1 \%$ & $19,0 \%$ & $11,0 \%$ & $100 \%$ \\
\hline Île-de-France & $67,9 \%$ & $4,3 \%$ & $1,3 \%$ & $14,8 \%$ & $11,8 \%$ & $100 \%$ \\
\hline Ouest & $62,3 \%$ & $3,9 \%$ & $3,4 \%$ & $15,6 \%$ & $14,8 \%$ & $100 \%$ \\
\hline Sud-Ouest & $70,6 \%$ & $2,5 \%$ & $3,6 \%$ & $14,6 \%$ & $8,7 \%$ & $100 \%$ \\
\hline Sud-Est & $68,1 \%$ & $3,8 \%$ & $1,5 \%$ & $14,3 \%$ & $12,3 \%$ & $100 \%$ \\
\hline Total & $67,1 \%$ & $3,5 \%$ & $2,5 \%$ & $15,0 \%$ & $12,0 \%$ & $100 \%$ \\
\hline
\end{tabular}

étant sous-représentés de façon significative : $32 \%$ des enfants habitant dans le Nord de la France jouent au rugby à l'école contre $37,4 \%$ dans le Sud. De même à l'échelle des cinq zones géographiques, $52,1 \%$ des jeunes interrogés de la «zone SudOuest » font du rugby à l'école contre 28,4\% des jeunes rugbymen de la « zone ouest » et $32,6 \%$ des jeunes enquêtés de la « zone Île-de-France » (voir tableau 5). Le construit social et les représentations qui se rattachent à la pratique du rugby semblent instituer la pratique à l'école. La disparité géographique du monde rugbystique est, dans le cadre de notre recherche, visible à travers l'institution scolaire. Ainsi, chez les jeunes joueurs de 9 à 11 ans interrogés, l'école est une institution exerçant une influence supérieure dans le Sud-Ouest.

\begin{tabular}{|c|c|c|c|}
\hline Joues-tu au rugby à l'école? & Oui & Non & Total \\
\hline Nord & $38,0 \%$ & $62,0 \%$ & $100 \%$ \\
\hline Île-de-France & $32,6 \%$ & $67,4 \%$ & $100 \%$ \\
\hline Ouest & $28,4 \%$ & $71,6 \%$ & $100 \%$ \\
\hline Sud-Ouest & $52,1 \%$ & $47,9 \%$ & $100 \%$ \\
\hline Sud-Est & $31,9 \%$ & $68,1 \%$ & $100 \%$ \\
\hline Total & $35,2 \%$ & $64,8 \%$ & $100 \%$ \\
\hline
\end{tabular}

Tableau 5 : La pratique du rugby à l'école, selon l'appartenance régionale $(\mathrm{p}<0.05)$ $(p<0.05)$

Rugby played at school, according to geographical areas

Ainsi, quelques différences régionales sont retrouvées dans l'accès à l'ensemble de la pratique sportive, mais excepté pour l'école, elles ne permettent pas de mettre en avant une distinction nette entre les zones géographiques dites rugbystiques ou non. Dès 1990, Augustin (1990, p. 97) écrivait que, si le rugby reste le vecteur d'une identité régionale, « certains pensent qu'il faut distinguer les plans symboliques de ces faits bruts [...] et que, en sport, les gens du Sud-Ouest ont le rugby au cœur et le football en tête ». Les résultats mettent en avant la construction sociale du monde rugbystique car, à l'échelle de notre échantillon, les différences culturelles ne sont principalement pas significatives en fonction des régions. Dans ce sens, nos résultats discutent la question d'une identité régionale affirmée dans l'accès à la pratique des jeunes joueurs, c'est-à-dire dans ce que Augustin (1990) qualifie de faits bruts. En effet, nous montrons que très peu de différences régionales ressurgissent quant aux conditions d'entrée des joueurs âgés de 9 à 11 ans dans le rugby. Ce qui, par ailleurs, ne contredit pas une possible identification symbolique de la communauté rugbystique à certains territoires (Augustin, Garrigou, 1985). Les résultats renforcent l'idée selon laquelle le monde rugbystique est une construction sociale, entre autres permise par un univers de discours " constitué par un groupe d'individus qui participent, en le réalisant, à un processus social d'expérience et de conduite, au sein duquel ces gestes et ces symboles ont une signification commune pour tous les membres du groupe - qu'ils les produisent et les adressent à d'autres individus ou qu'ils y répondent en tant qu'ils leur sont adressés par d'autres individus » (Mead, 1934, p. 89-90). Ainsi, le monde de l'ovalie est un monde social dynamique, une entité active (Bodin et Héas, 2002), qui se définit par l'activité des joueurs et entraîneurs, des journalistes et commentateurs spécialisés, des spectateurs et supporters. La territorialité y est pérennisée par des conventions (Becker, 1988) créatrices de liens entre les acteurs qui vont se sentir appartenir au même monde : expressions emblématiques, accent, mode vestimentaire, style de vie, esprit de camaraderie, comme l'écrit Tonini (2007, p. 20) : « à force de présence, le sport imprègne les mentalités et devient, par là un élément d'identité, voire de culture locale». 


\section{Conclusion}

Nous pensions que le monde du rugby serait davantage visible dans les caractéristiques de la socialisation sportive des jeunes joueurs. En réalité, cela n'a pas été perçu - tout au moins par l'intermédiaire des outils choisis dans le cadre de cette enquête. Les résultats font plutôt émerger des similarités à l'échelle nationale.

Pour autant, la régionalisation du rugby n'est pas remise en question : l'effet régional sur la répartition des licenciés ne peut pas être contesté. Ainsi, en valeur absolue, les jeunes joueurs de rugby sont plus nombreux dans les régions du Sud de la France que dans celles du Nord. Toutefois, paradoxalement, les résultats tendent à montrer l'absence d'une spécificité territoriale dans l'accès à la pratique, pourtant réputée particulièrement régionale. Ainsi, il apparaît que l'entrée des jeunes de 9 à 11 ans dans le monde de l'ovalie est régionalement assez peu différenciée. Ces résultats questionnent donc la pertinence de l'idée d'une identité sportive territoriale de la pratique du rugby. Si des différences régionales mineures apparaissent, aucune région ne se distingue réellement car aucune ne s'écarte de la tendance majoritaire quant aux conditions d'accès des joueurs à la pratique. Seule l'institution scolaire semble conserver une influence différenciée. Ces différences inter-régionales relevées à propos de l'influence de l'école sont certes significatives, mais somme toute assez anecdotiques car l'école reste de manière générale peu influente comme facteur de socialisation rugbystique.

Des analyses complémentaires envers d'autres catégories d'âge seraient utiles pour répondre de manière plus complète à la question d'une spécificité de certaines régions du Sud-Ouest. D'autant plus que les résultats de ce travail peuvent s'expliquer, chez ces jeunes joueurs, par l'influence importante de la famille dans le choix d'une activité et des effets de surdétermination liés à l'offre des clubs de rugby. Il serait intéressant de poursuivre ce travail auprès des joueurs âgés de plus de 11 ans, chez qui l'importance territoriale est possible, notamment par l'intermédiaire du sport professionnel à travers l'influence des grands clubs et le marketing associé ainsi que la socialisation par groupe de pairs qui agit souvent dans le choix des pratiques culturelles à cet âge.

\section{Bibliographie}

Appadurai A., 2001. Après le colonialisme. Les conséquences culturelles de la globalisation, Paris, Payot, coll. «Petite Bibliothèque Payot », 336 p.

Augustin J.-P., 1990. La percée du football en terre de rugby. L'exemple du sud-ouest français et de l'agglomération bordelaise, Vingtième Siècle. Revue d'histoire, n 26, p. 97-110.

Augustin J.-P., 1 995. Sport, Géographie et Aménagement, Nathan Université, Paris, 254 p.

Augustin J.-P., 2004. Le rugby : une culture monde territorialisée, Outre-Terre, no 8/3, p. 261-273. DOI : 10.3917/ oute.008.0261.

Augustin J.-P., 2007. Géographie du sport, Spatialités contemporaines et mondialisation, Paris, Armand Colin, 224 p.

Augustin J.-P., Bodis J.-P., 1994. Rugby en Aquitaine, histoire d'une rencontre, Bordeaux, Auberon et CLRA, 315 p.

Augustin J.-P., Garrigou A., 1985. Le rugby démêlé, Bordeaux, Le Mascaret, $360 \mathrm{p}$.

Augustin J.-P., Joncheray H., 2007. Le rugby en Australie et en France : des diffusions et des implantations différenciées, in Guillain J.-Y., Porte P. (dir.) La planète est rugby, regards croisés sur l'Ovalie, Biarritz, Atlantica, p. 161-183.

Becker H. S., 1988. Les mondes de l'art, Paris, Flammarion, $382 \mathrm{p}$.

Becker H. S., Pessin A., 2006. Dialogue sur les notions de Monde et de Champ, Sociologie de l'Art, no 1, vol. 8, p. 163180. DOI : 10.3917/soart.008.0163.

Bodin D., HÉAs S., 2002. Introduction à la sociologie des sports, Paris, Chiron, 252 p.

Bois J., Sarrazin P., 2006. Les chiens font-ils des chats? Une revue de littérature sur le rôle des parents dans la socialisation de leur enfant pour le sport, Science $E$ Motricité, n ${ }^{\circ} 57$, vol. 1, p. 9-54.

Brustad R. J., Babkes M. L., Smith A. L., 2001. Youth in sport: Psychological considerations, in Singer R. N., Hausenblas H. A., Janelle C. M. (dir.), Handbook of Sport Psychology, New York, John Wiley, p. 604-635.

Brustad R. J., 1993. Who Will Go Out and Play? Parental and Psychological Influences on Children's Attraction to Physical Activity, Pediatric Exercice Science, $n^{\circ} 5$, vol. 3, p. 210-223.

Callède J.-P., 1996. Implantation, diffusion et rayonnement du rugby dans la France du Sud, in Sagnes J. (dir.), Le Sport dans la France contemporaine, Perpignan, Presses universitaires de Perpignan, p. 67-104.

Callède J.-P., 1993. Histoire du sport en France du Stade Bordelais au SBUC, 1889-1939, Éditions de la Maison des Sciences de l'homme d'Aquitaine, Bordeaux, 211 p.

Coakley J., Pike E., 2009. Sports in Society: Issues and Controversies, McGraw-Hill Higher Education, European Edition, $613 \mathrm{p}$.

Coakley J., White A., 1992. Making decisions: gender and sport participation among British adolescents, Sociology of Sport Journal, no 9, p. 20-35. DOI: 10.1123/ssj.9.1.20.

Darbon S., 1997. La « grande famille » du rugby, Communications, $\mathrm{n}^{\circ} 65$, p. 49-57. DOI : 10.3406/comm.1997.1986. 
Fuchs J., Augustin J.-P., 2014. Des sports et des jeunes, dossier coordonné par J. Fuchs et J.-P. Augustin, Agora débats / jeunesse, Paris, Presses de Sciences Po, n 68, vol. 3.

GaY J.-C., 2006. Sur les pistes de la mondialisation, Mappemonde, $\mathrm{n}^{\circ} 82$, vol. 2.

Haumont A., 1995. Les variations géographiques du sport, in Sport, relations sociales et action collective, Actes du colloque des 14 et 15 octobre 1993, Bordeaux/Talence, Éditions Maison des sciences de l'Homme d'Aquitaine, p. 51-59.

Joncheray H., Level M., Richard R., 2016. Socialization and construction of identity(ies) of the players of the French national rugby union women's team, International Review for the Sociology of Sport, $\mathrm{n}^{\circ}$ 51, p. 162-177. DOI: 10.1177/1012690213517108.

Joncheray H., Laporte R., Tenèze L., 2015. Analyse des processus d'engagement et de désengagement dans une pratique sportive. Le cas des adolescents français joueurs de rugby, Loisir et Société/Society and Leisure, $\mathrm{n}^{\circ} 38$, vol. 3, p. 436-456. DOI: 10.1080/07053436.2015.1083759.

Joncheray H., Thili H., 2013. Are there still Social Barriers to Women's Rugby?, Sport in Society, no 16, vol. 6, p. 772-788.

Lacouture J., 1993. Voyous et gentlemen. Une histoire du rugby, Paris, Gallimard, $176 \mathrm{p}$.

Lewko J. H., Greendorfer S. L., 1988. Family influences in sport socialization of children and adolescents, in SMOLL F., Magill R., And Ash M., Children in Sport, Champaign, Human Kinetics, p. 287-300.

Manale M., 2007. Vers un nouvel horizon indépassable? Identités et Territoires au $\mathrm{xxI}^{\mathrm{e}}$ siècle, L'Homme et la société, $\mathrm{n}^{\circ} 3$, vol. 165-166, p. 11-15.

Mathieu D., Praicheux J., 1987. Sports en France, Paris, Fayard-Reclus, $120 \mathrm{p}$.

Mathieu D., Praicheux J., Volle J.-P., 1992. Sports en France, Mappemonde, $\mathrm{n}^{\circ}$ 2, p. 2-5.

McPherson Brown T., 1988. The structure, processes, and consequences of sport for children, in SMOLl F., Magill R.,
Ash M., Children in Sport, Champaign, Human Kinetics, p. 265-286.

Mead G. H., 1934. L'Esprit, le soi et la société, Paris, PUF, $428 \mathrm{p}$.

Mennesson C., 2005. Etre une femme dans le monde des hommes. Socialisation sportive et construction du genre, Paris, L'Harmattan, $365 \mathrm{p}$.

Ravenel L., 2004. Pourquoi n'y a-t-il pas de rugby en Normandie? Un essai d'explication géographique, Norois, n 190 , p. 85-95. DOI : 10.4000/norois.79.

Ravenel L., 2000. La création du « National » : une décision qui a modifié la structure géographique du football professionnel français ", Géopoint 98 : Décision et analyse spatiale, p. 232-235.

Ravenel L., 1998. La Géographie du football en France, Paris, PUF, coll. «Pratiques Corporelles », 143 p.

Sмiтн R., Smoll F., 1978. Sport and the child: Conceptual and research perspectives, in Smoll F., Smith R., Psychological perspectives in youth sports, Washington, Hemisphere.

Stat-info, 2014. Bulletin de statistiques et d'études, Ministère des Droits des Femmes, de la Ville, de la Jeunesse et des Sports, $n^{\circ} 14$, vol. 1 .

STrauss A., 1978. Negotiations. Varieties, contexts, processes and social order, San Francisco, Jossey-Bass publishers, 270 p.

Tonini B., 2007. La dynamique spatiale des pratiques sportives. Des patronages à l'intercommunalité. L'exemple du basketball, du football et du rugby dans les Pays de la Loire. Thèse de doctorat en géographie, Université de Nantes, 339 p.

Wahl A., 1989. Les Archives du football: sport et société en France (1880-1980), Paris, Gallimard/Julliard, coll. « Archives », 355 p.

Young K., 1999. Sporting Bodies, Damaged Selves: Sociological Studies of Sports-Related Injury, Oxford, Elsevier Press, 396 p. 